\title{
0 autoconceito de estudantes aceitos e rejeitados no contexto escolar
}

\author{
The self-concept of students accepted and rejected within the school environment
}

Andreza Schiavoni ${ }^{[a]}$, Selma de Cássia Martinellif ${ }^{[b]}$

${ }^{[a]}$ Doutora na área de Psicologia Educacional pela Universidade Estadual de Campinas (Unicamp), Campinas, SP - Brasil, e-mail: andrezaschiavoni@hotmail.com

${ }^{[b]}$ Professora Doutora do Departamento de Psicologia Educacional da Universidade Estadual de Campinas (Unicamp), Campinas, SP - Brasil, e-mail: selmacm@unicamp.br

Recebido: $14 / 10 / 2010$ Received: 10/14/2010

Aprovado: 10/11/2010 Approved: $11 / 10 / 2010$

\section{Resumo}

A presente pesquisa teve como objetivo investigar se havia diferenças no autoconceito de crianças que apresentavam diferentes padrões de aceitação e rejeição por parte dos amigos da escola. Participaram do estudo 207 crianças entre 7 e 11 anos de idade, de duas escolas públicas do Estado de São Paulo. Para avaliar o status sociométrico foi solicitado aos participantes que indicassem três nomes de colegas da classe com quem gostariam de estudar, de brincar, de não estudar e de não brincar. 0 autoconceito foi obtido por meio de uma escala contendo 20 afirmações que buscam a avaliar o autoconceito social, escolar, familiar e pessoal dos participantes. A amostra ficou dividida em grupos distintos: o grupo dos isolados, que foi representado por aqueles estudantes que não foram lembrados nenhuma vez por seus colegas de sala; um grupo com alta aceitação e alta rejeição; um grupo com aceitação e rejeição moderadas; e um grupo com baixa aceitação e baixa rejeição. Os resultados indicaram diferenças no autoconceito escolar em meio aos grupos de aceitação entre os colegas. Os estudantes isolados e aqueles com aceitação moderada mostraram um autoconceito escolar mais positivo do que os alunos com baixa aceitação. No que diz respeito à rejeição, observou-se que os estudantes com baixa rejeição no grupo de amigos mostraram ter um autoconceito familiar mais positivo do que aqueles com moderada e alta rejeição. Verificou-se também que os alunos isolados revelaram um autoconceito pessoal mais elevado do que aqueles com rejeição moderada e alta entre os colegas.

Palavras-chave: Autoconceito. Status sociométrico. Relacionamento interpessoal.

\section{Abstract}

This study aimed to investigate whether there were differences in self-concept of children who were faced with different standards of acceptance and rejection by their classmates. Participants were 207 children aged between 7 and 11 years old from two public schools of São Paulo State. Participants were required to indicate three classmates with whom they would like to study and to play, and three with whom they would not like to study and to play, as to assess the sociometric status. Self-concept was measured through a scale containing twenty statements that evaluate the social, academic, family and personal participants' self-concept. The sample was divided into 
distinct groups: the socially isolated group, which was represented by those students who were not appointed by their classmates; a group with high acceptance and high rejection; a group with moderate acceptance and rejection; and a group with low acceptance and low rejection. The results indicated differences in academic self-concept among the groups of peer acceptance. The isolated students and those with moderate acceptance showed a more positive academic selfconcept than students with low acceptance. Regarding rejection, it was observed that pupils with low rejection among the group of students have presented a more positive family self-concept than those with moderate to high rejection. It was also found that the isolated students showed a higher personal self-concept than those with moderate and high rejection among colleagues.

Keywords: Self-concept. Sociometric status. Interpersonal relationship.

\section{Introducãa}

A vida de um indivíduo é continuamente influenciada por diferentes aspectos, sejam eles sociais, familiares, educacionais, entre muitos outros. No período em que o sujeito frequenta a escola, muitos aspectos o estão influenciando e por ele sendo influenciados. Tem-se reconhecido, na literatura, a importância das variáveis afetivas na vida acadêmica de alunos, no seu desempenho e nas dificuldades experimentadas no seu processo de aprendizagem, bem como nas relações estabelecidas com os outros. Dentre elas, o autoconceito tem sido investigado, e os estudos têm apontado para a importância desse constructo na vida das pessoas, tanto em sua vida pessoal como familiar, social e acadêmica.

De acordo com Sisto e Martinelli (2004), tem-se observado um grande interesse de pesquisadores em investigar o autoconceito de estudantes, com o intuito de entender a importância dessa variável na vida do aluno, o que pode ser uma fonte de informação para explicar muitos dos problemas que estes vivenciam. No estudo do autoconceito, segundo os autores, a literatura tem apontado um certo consenso entre os pesquisadores quanto a três aspectos: um deles é o caráter desenvolvimental do autoconceito, já que se entende que este não nasce com o indivíduo, mas é construído desde a infância e ao longo da vida, e é desenvolvido com base na relação com o outro. Assim, o reconhecimento da influência social constitui o segundo aspecto consensual. A ideia de multidimensionalidade, terceiro aspecto, é sustentada pela crença na existência de vários "eus", construídos de acordo com o contexto social, sendo, então, possível falar de um autoconceito familiar, baseado e desenvolvido nas relações com os familiares, um autoconceito escolar, estabelecido com base nas interações com os professores e colegas, entre outros.

A análise do contexto familiar revela que este é marcado por interações e situações que influenciam na percepção que o indivíduo vai construindo de si. Estudos têm sido realizados para verificar a relação entre o autoconceito dos filhos e o ambiente famíliar. Magagnin e Körbes (2000), por exemplo, investigaram a relação entre o autoconceito do adolescente e o relacionamento familiar com pais e irmãos. Os resultados mostraram que altos escores de autoconceito se relacionaram a um bom relacionamento com pais e irmãos, enquanto baixos escores de autoconceito se relacionaram a um relacionamento conflituoso. Resultados semelhantes foram encontrados por Wentzel e Feldman (1996), Sánchez e Escribano (1999).

As pesquisas que se preocupam em investigar a relação entre desempenho acadêmico e autoconceito também têm crescido nas últimas décadas, principalmente por se considerar que, no contexto escolar, muitas são as interações e as variáveis que podem contribuir para a construção de uma autopercepção boa ou ruim. Jacob (2001) encontrou correlação entre o desempenho escolar e autoconceito e autoestima em crianças do ensino fundamental. Urquijo (2002) investigou a relação entre autoconceito e desempenho acadêmico em linguagem e matemática de adolescentes. Os dados mostraram correlações positivas e significativas entre autoconceito e desempenho para as duas áreas. Trabalhos mais atuais têm apontado para a mesma direção. Resultados semelhantes foram encontrados por Suehiro (2006), 
que avaliou a relação desempenho acadêmico e autoconceito entre alunos do ensino superior.

A preocupação em investigar a relação entre autoconceito e dificuldades de aprendizagem também pode ser notada. A maioria dos resultados sugere que escores mais baixos de autoconceito são encontrados para os alunos que apresentam dificuldades, se comparados àqueles que não apresentam (Cooley \& Ayres, 1988; Durrant, Cunningham \& Volker, 1990; Rothman \& Cosden, 1995). Mais recentemente, pode-se encontrar o trabalho de Carneiro, Martinelli e Sisto (2003) que verificaram as diferenças no autoconceito geral, escolar, social, familiar e pessoal, em grupos de crianças que apresentavam diferentes níveis de dificuldades na escrita, e encontraram uma relação significativa entre as dificuldades em escrita e o autoconceito geral e escolar. Okano, Loureiro e Linhares (2004) também investigaram os escores de autoconceito entre crianças de dois grupos, um composto por alunos que apresentavam dificuldades de aprendizagem e outro por crianças que não apresentavam nenhuma dificuldade. Os resultados mostraram escores significativamente menores entre as crianças do primeiro grupo.

Os estudos sobre o autoconceito indicam claramente a forte influência exercida pelo outro e pelo meio social na vida pessoal dos indivíduos. 0 reconhecimento da importância da vida social e do quanto esta interfere na construção de si mesmo nos remete à importância atribuída por alguns teóricos à participação e à inserção do sujeito em um grupo. Por este motivo, estudos têm se preocupado em identificar a posição sociométrica que as pessoas ocupam nos grupos aos quais pertencem, visto que, para participar adequadamente de um grupo, é exigido um grau de aceitação por parte de seus membros (Newcombe, 1999).

No contexto escolar, pode-se observar que alguns alunos são mais aceitos que outros. Geralmente esses são os que demonstram grande número de competências cognitivas e sociais, revelando comportamentos amigáveis e afetuosos, além de compreenderem e praticarem formas eficazes de interação. Por outro lado, alunos menos aceitos por seus pares geralmente apresentam um padrão de comportamento, que se caracteriza por incluir a promoção de conflitos, provocações, brigas, discussões e agressões, além de imaturidade e habilidades sociais e cognitivas reduzidas (Newcombe, 1999).
Segundo a pesquisa realizada por Silva e Lohr (2001), as crianças de $4^{\text {a }}$ série investigadas tenderam a rejeitar os seus pares com base em comportamentos inadequados por eles emitidos, como autoritarismo e conduta perturbadora. Em contrapartida, o que levou às escolhas de seus pares foram os comportamentos pró-sociais emitidos. Essa percepção também foi verificada por parte dos professores que participaram do estudo.

A preocupação em investigar o status sociométrico de estudantes e suas relações com a aprendizagem escolar também tem sido frequente. Sisto, Urquijo e Souza (1999) investigaram as relações entre desenvolvimento cognitivo medido por provas de imagem mental, provas operatórias de conservação de massa e comprimento, construção de possíveis em equidistância e arranjos de dados e o teste sociométrico. Os resultados indicaram que crianças com alta aceitação por seus pares apresentaram um desenvolvimento cognitivo geral superior ao das crianças com alta rejeição.

Lopes, Cruz e Rutherford (2002) avaliaram alunos portugueses de $5^{\underline{a}}$ e $6^{\underline{a}}$ séries considerados como rejeitados e negligenciados e encontraram que esses foram os que pior desempenho apresentaram, além de demonstrarem mais comportamentos agressivos com os colegas e mais problemas com os professores.

Stevanato, Loureiro, Linhares e Marturano (2003) verificaram o autoconceito de alunos sem dificuldades e com dificuldades de aprendizagem, sendo que alguns deles também apresentavam problemas de comportamento. Observou-se que o autoconceito se mostrou mais negativo nos escores global e específico para os alunos com dificuldades de aprendizagem. Nenhuma diferença foi encontrada entre as crianças com dificuldade de aprendizagem diferenciadas quanto à presença de problemas de comportamento.

Saravali (2004) investigou a posição sociométrica que alunos, apontados pela professora como apresentando dificuldades de aprendizagem, ocupavam no grupo ao qual pertenciam. Avaliou-se também a relação entre a posição sociométrica e os dados obtidos pela análise do histórico escolar desses alunos. Os resultados apontaram para a baixa ou nenhuma escolha por parte dos colegas para as crianças com queixa de dificuldade de aprendizagem. Observouse, ainda, que o julgamento da professora com relação ao aluno com dificuldades de aprendizagem tem relação direta com o número de escolhas que ele recebe no teste sociométrico. Todas as crianças 
apontadas pela professora foram pouco escolhidas nos critérios afetivo e intelectual. Verificou-se, por fim, que as crianças que tinham os piores conceitos no histórico escolar tiveram também menos escolhas no teste do que as outras crianças com dificuldade de aprendizagem que tinham conceitos um pouco melhores.

No que se refere ao estudo da avaliação sociométrica de alunos, observa-se, também, que há uma preocupação em relacioná-la a outras variáveis, além da aprendizagem. Pode-se notar a presença de trabalhos que relacionam o status sociométrico a características comportamentais (Lee-Manoel, Morais, Bussab \& Otta, 2002; Morais, Otta \& Scala, 2001); à agressividade (Coie, Terry, Lenox, Lochman \& Hyman, 1995; Sisto, 2003; Sisto, 2005); a problemas de atenção (Toneloto, 2002); às percepções da criança com respeito a sua interação com o professor (Martinelli \& Schiavoni, 2009), entre outras.

Poucos trabalhos têm se dedicado a estudar a relação entre o status sociométrico de alunos e o autoconceito. Nenhum estudo que pesquisou essa relação foi encontrado no Brasil. Na literatura internacional, observa-se a existência dos estudos de Rudolph (1995) e de Jackson e Bracken (1998).

Rudolph (1995) examinou como as representações de si e do outro, entre crianças de sete e doze anos, podem funcionar como mediadoras nas relações com os pares e com os familiares. Os resultados indicaram que percepções negativas de si e de outros se relacionaram a um desajustamento social, incluindo um status menos positivo no grupo. Jackson e Bracken (1998) avaliaram o autoconceito global e específico de crianças e adolescentes considerados pelo status social como populares, rejeitados, negligenciados, controversos, medianos e indiferentes. Os achados sustentaram a hipótese de que os alunos pertencentes a grupos extremos diferem no autoconceito.

Os estudos apresentados reforçam a ideia de que o desenvolvimento afetivo e social caminham juntos, e que conhecer mais acerca desse funcionamento ou de suas relações é primordial para que se possa também compreender de forma mais ampla a dinâmica das ligações que se estabelecem entre os aspectos cognitivos, afetivos e sociais. Pensando nessas relações verificadas e reveladas por essas pesquisas é que Sisto e Martinelli (2006) pontuaram que a criança que apresenta dificuldades em desempenhar bem suas funções sociais tende a sofrer consequências emocionais que podem levar a uma baixa autoestima ou mesmo a uma percepção negativa de si mesmo. Por outro lado, a criança que se percebe como não querida ou adequada pode, por sua vez, adotar um padrão de comportamento que altere suas relações com seus companheiros. Parece certo, então, dizer da importância de avaliar essas relações. Por este motivo, o objetivo deste estudo foi verificar se havia diferenças no autoconceito de crianças que apresentavam diferentes padrões de aceitação e rejeição pelos amigos da escola.

\section{Método}

\section{Participantes}

Participaram desta pesquisa 207 alunos entre 7 e 11 anos de idade, sendo 103 do sexo masculino $(49,8 \%)$ e 104 do sexo feminino (50,2\%), que frequentavam o terceiro, quarto e quinto anos do ensino fundamental de duas escolas públicas estaduais de dois municípios do Estado de São Paulo. Os participantes foram avaliados quanto à sua aceitação e rejeição no grupo de amigos, por meio da técnica sociométrica e com relação ao seu autoconceito. A partir da pontuação bruta obtida foi possível dividir a amostra em grupos distintos tanto para a aceitação quanto para a rejeição. 0 grupo dos isolados, que foi representado por aqueles estudantes que não foram lembrados nenhuma vez por seus colegas de sala; um grupo com alta aceitação e alta rejeição; um grupo com aceitação e rejeição moderadas; e um grupo com baixa aceitação e baixa rejeição.

\section{Instrumentos}

Medida de avaliação sociométrica. Trata-se de um instrumento com quatro questões, duas referentes a estudar e duas relativas a brincar. Nelas, os participantes deveriam indicar três nomes de colegas da classe com quem gostariam de estudar, três com quem não gostariam de estudar, três com quem gostariam de brincar e mais três com quem não gostariam de brincar. Para as questões referentes à aceitação (estudar e brincar) foram atribuídas as pontuações 3 para a primeira escolha, 2 para a segunda e 1 para a terceira. Os mesmos valores foram atribuídos às questões relativas à rejeição (não estudar e não brincar), sendo, então, 3 para a primeira escolha, 2 para a segunda e 1 para a terceira. Uma 
soma dos valores foi feita para cada participante, 0 que resultou em uma medida de aceitação e outra de rejeição. Essa avaliação foi usada para agrupar os participantes nos quatro diferentes grupos de aceitação e rejeição.

Escala de autoconceito infanto-juvenil, EAC-IJ (Sisto \& Martinelli, 2004). Trata-se de uma escala que contém 20 afirmações, distribuídas em: seis relativas ao autoconceito social, que se referem às percepções a respeito das relações sociais com os colegas; cinco relativas ao autoconceito escolar, que revelam os sentimentos do indivíduo referentes às relações interpessoais na escola e às percepções sobre ser esperto, bondoso e divertido; quatro afirmações acerca do autoconceito familiar, que indicam a avaliação com relação às situações cotidianas em casa com os pais e os irmãos; e cinco relativas ao autoconceito pessoal, que tratam das percepções a respeito de sua maneira de agir em diferentes situações, medo, erros, preocupação, nervosismo. Para as escalas de autoconceito pessoal e social as opções de resposta sempre, às vezes e nunca foram pontuadas com 0,1 e 2 pontos, respectivamente, enquanto que para as escalas escolar e familiar foram atribuídas as pontuações 2, 1 e 0 para as mesmas opções de respostas.

\section{Procedimento de coleta de dados}

Após o consentimento da equipe gestora e dos professores, foi enviada aos pais uma carta na qual eram orientados a respeito dos objetivos do estudo, do tipo de participação requerida, bem como da ausência de prejuízos decorrentes desta. Somente foram convocados a responderem os instrumentos os alunos autorizados pelos pais a participarem da pesquisa mediante assinatura de um termo de consentimento livre e esclarecido. A aplicação dos instrumentos ocorreu de forma coletiva, realizada nas salas de aulas dos alunos e somente àqueles cujos pais haviam autorizado. Inicialmente foi explicado às crianças de que se tratava a pesquisa e como responder aos instrumentos.

\section{Resultados}

Após diferenciar os grupos em função de seu grau de aceitação e de rejeição, foi feita uma comparação em relação às diferentes dimensões de seu autoconceito, sendo elas social, escolar, familiar e pessoal. Na Tabela 1 encontram-se discriminadas as diferenças de média dos grupos de aceitação em relação ao autoconceito da criança.

Tabela 1 - Médias de autoconceito em função dos grupos de aceitação

\begin{tabular}{cccccc}
\hline Aceitação & N & $\begin{array}{c}\text { Auto- } \\
\text { conceito } \\
\text { Social }\end{array}$ & $\begin{array}{c}\text { Auto- } \\
\text { conceito } \\
\text { Escolar }\end{array}$ & $\begin{array}{c}\text { Auto- } \\
\text { conceito } \\
\text { Familiar }\end{array}$ & $\begin{array}{c}\text { Auto- } \\
\text { conceito } \\
\text { Pessoal }\end{array}$ \\
\hline $\begin{array}{c}\text { Grupo1- } \\
\text { Isolados }\end{array}$ & 33 & 6,97 & 5,70 & 6,64 & 7,06 \\
$\begin{array}{c}\text { Grupo2- } \\
\text { Baixa Aceita- } \\
\text { ção }\end{array}$ & 44 & 7,39 & 4,77 & 6,39 & 6,36 \\
$\begin{array}{c}\text { Grupo3- } \\
\text { Aceitação }\end{array}$ & 83 & 7,84 & 5,60 & 6,28 & 6,35 \\
Moderada & & & & & \\
$\begin{array}{c}\text { Grupo4- } \\
\text { Alta Aceitação }\end{array}$ & 47 & 8,02 & 5,17 & 6,68 & 7,02 \\
\hline \begin{tabular}{c} 
Total \\
\hline
\end{tabular} & 207 & & & & \\
\hline
\end{tabular}

Fonte: Dados da pesquisa.

Na medida de aceitação dos participantes, encontraram-se pontuações que variaram de 0 a 47, indicando que algumas crianças não foram nenhuma vez escolhidas para brincar e para estudar por nenhum dos colegas, enquanto outras foram escolhidas por vários deles. Os dados apontados na Tabela 1 indicam que grande parte dos estudantes $(\mathrm{N}=83$, que representam 40,09\% da amostra) apresentou uma aceitação moderada no grupo de amigos. Do restante, 44 participantes $(21,25 \%)$ apresentaram baixa aceitação, 47 (22,70\%), alta aceitação, e 33 $(15,94 \%)$ foram considerados isolados.

Para verificar se existiam diferenças entre os grupos no que diz respeito ao autoconceito, foi aplicado o teste de Kruskall-Wallis. Tal teste revelou não existirem diferenças estatisticamente significantes entre os grupos no que se refere ao autoconceito social ( $p=0,229)$, familiar $(p=0,326)$ e pessoal $(p=0,246)$. Por outro lado, observou-se que para os grupos de aceitação social foi encontrado um valor de $\mathrm{p}=0,055$ para o autoconceito escolar, que se aproxima muito do grau de significância estatística e que, portanto, será considerado nas análises que se seguem.

Com o objetivo de conhecer a direção dessa diferença observada no autoconceito escolar, entre os grupos de aceitação, aplicou-se o teste de Mann-Whitney para amostras independentes, como aponta a Tabela 2. 
Tabela 2 - Incidência média, valores de U, níveis de significância e comparação entre os diferentes grupos de aceitação entre os seus pares e seu autoconceito escolar

\begin{tabular}{ccccc}
\hline $\begin{array}{c}\text { Grupos pelo } \\
\text { Critério de } \\
\text { Rejeição }\end{array}$ & $\begin{array}{c}\text { Valores } \\
\text { de U }\end{array}$ & $\mathbf{Z}$ & $\begin{array}{c}\text { Valores } \\
\text { de } \mathbf{p}\end{array}$ & $\begin{array}{c}\text { Comparação } \\
\text { entre os } \\
\text { Grupos }\end{array}$ \\
\hline G1 e G2 & 501,500 & $-2,353$ & 0,019 & G1>G2 \\
G1 e G3 & 1313,000 & $-0,351$ & 0,726 & SD* \\
\hline G1 e G4 & 613,500 & $-1,612$ & 0,107 & SD \\
\hline G2 e G3 & 1386,500 & $-2,254$ & 0,024 & G2<G3 \\
\hline G2 e G4 & 914,500 & $-0,963$ & 0,335 & SD \\
\hline G3 e G4 & 1699,500 & $-1,231$ & 0,218 & SD \\
\hline
\end{tabular}

*SD: Sem Diferenças Estatisticamente Significantes Fonte: Dados da pesquisa.

Esses resultados indicam uma diferença estatisticamente significante entre os grupos 1 e 2, sugerindo que o grupo de estudantes isolados, ou seja, aqueles que não foram escolhidos por seus colegas, apresentou um autoconceito escolar mais elevado do que os alunos com baixa aceitação entre os seus pares. Além disso, os dados apontaram significância também na diferença entre os grupos 2 e 3 , indicando que os participantes pertencentes ao grupo de aceitação moderada mostraram um autoconceito escolar mais positivo do que aqueles com baixa aceitação no grupo de amigos. 0 dado apresentado na Tabela 1 permite ainda verificar que o grupo de crianças isoladas foi o que teve a maior média no autoconceito escolar quando comparado aos demais grupos. Considerando que a subescala de autoconceito escolar avalia a percepção do sujeito quanto à sua liderança, ao se considerar esperto, bondoso e ouvido pelos colegas, o que se observa é que os estudantes que não foram lembrados pelos seus colegas foram os que mais manifestaram perceber-se com essas características ou, por outro lado, podem também ter respondido em função de um desejo de serem vistos dessa forma.

No que se refere à rejeição no grupo de amigos, encontram-se discriminadas, na Tabela 3 , as diferenças de média em função do autoconceito da criança.
Tabela 3 - Médias de autoconceito em funç̃o dos grupos de rejeição

\begin{tabular}{cccccc}
\hline Rejeição & N & $\begin{array}{c}\text { Auto- } \\
\text { conceito } \\
\text { Social }\end{array}$ & $\begin{array}{c}\text { Auto- } \\
\text { conceito } \\
\text { Escolar }\end{array}$ & $\begin{array}{c}\text { Auto- } \\
\text { conceito } \\
\text { Familiar }\end{array}$ & $\begin{array}{c}\text { Auto- } \\
\text { conceito } \\
\text { Pessoal }\end{array}$ \\
\hline $\begin{array}{c}\text { Grupo1- } \\
\text { Isolados }\end{array}$ & 39 & 8,10 & 4,90 & 6,67 & 7,59 \\
$\begin{array}{c}\text { Grupo2- } \\
\text { Baixa } \\
\text { Rejeição }\end{array}$ & 49 & 7,53 & 5,51 & 6,84 & 6,82 \\
$\begin{array}{c}\text { Grupo3- } \\
\text { Rejeição }\end{array}$ & 70 & 7,77 & 5,37 & 6,24 & 6,37 \\
Moderada & & & & & \\
\hline $\begin{array}{c}\text { Grupo4- } \\
\text { Alta Rejeição }\end{array}$ & 49 & 7,22 & 5,49 & 6,18 & 6,00 \\
\hline
\end{tabular}

Fonte: Dados da pesquisa.

Em relação à rejeição, pode-se notar que 70 alunos $(33,81 \%)$ foram indicados como aqueles com rejeição moderada entre os amigos, enquanto que $49(23,67 \%)$ deles se localizaram no grupo de baixa rejeição, 49 (23,67\%) no grupo de alta rejeição, e 39 $(18,84 \%)$ não foram escolhidos por nenhum dos colegas. Na medida de rejeição, as pontuações variaram de 0 a 53, indicando que, assim como que o verificado a medida de aceitação, enquanto algumas crianças não foram rejeitadas por seus colegas, outras foram lembradas com bastante frequência.

As diferenças de médias de autoconceito, encontradas entre os grupos com diferentes níveis de rejeição, analisadas pelo teste de Kruskall-Wallis, revelaram-se significantes no que diz respeito ao autoconceito familiar $(p=0,037)$ e pessoal $(p=0,007)$, e não significativas em relação ao autoconceito social $(p=0,321)$ e escolar $(p=0,455)$.

A direção dessas diferenças, verificadas pelo teste de Mann-Whitney, encontra-se discriminada na Tabela 4 , no que se refere ao autoconceito familiar, e na Tabela 5, no que diz respeito ao autoconceito pessoal. 
Tabela 4 - Incidência média, valores de U, níveis de significância e comparação entre os diferentes grupos de rejeição entre os seus pares e seu autoconceito familiar

\begin{tabular}{ccccc}
\hline $\begin{array}{c}\text { Grupos pelo } \\
\text { Critério de } \\
\text { Rejeição }\end{array}$ & $\begin{array}{c}\text { Valores } \\
\text { de U }\end{array}$ & $\mathbf{Z}$ & $\begin{array}{c}\text { Valores } \\
\text { de } \mathbf{p}\end{array}$ & $\begin{array}{c}\text { Comparação } \\
\text { entre os } \\
\text { Grupos }\end{array}$ \\
\hline G1 e G2 & 889,500 & $-1,568$ & 0,117 & SD* \\
G1 e G3 & 1135,000 & $-1,495$ & 0,135 & SD \\
\hline G1 e G4 & 758,500 & $-1,698$ & 0,089 & SD \\
\hline G2 e G3 & 1306,000 & $-2,282$ & 0,022 & G2 $>$ G3 \\
\hline G2 e G4 & 865,500 & $-2,456$ & 0,014 & G2 $>$ G4 \\
\hline G3 e G4 & 1652,000 & $-0,348$ & 0,728 & SD \\
\hline
\end{tabular}

*SD: Sem Diferenças Estatisticamente Significantes Fonte: Dados da pesquisa.

Esses resultados mostraram diferenças significantes entre os grupos G2-G3 e G2-G4, indicando que os estudantes com baixa rejeição entre os seus pares apresentaram um autoconceito familiar mais elevado do que aqueles dos grupos com rejeição moderada e com alta rejeição. Pode-se também observar, pelos resultados da Tabela 3, que o grupo com alta rejeição foi o que teve menor média na escala de autoconceito familiar.

Tabela 5 - Incidência média, valores de U, níveis de significância e comparação entre os diferentes grupos de rejeição entre os seus pares e seu autoconceito pessoal

\begin{tabular}{ccccc}
\hline $\begin{array}{c}\text { Grupos pelo } \\
\text { Critério de } \\
\text { Rejeição }\end{array}$ & $\begin{array}{c}\text { Valores } \\
\text { de } \mathbf{U}\end{array}$ & $\mathbf{Z}$ & $\begin{array}{c}\text { Valores } \\
\text { de } \mathbf{p}\end{array}$ & $\begin{array}{c}\text { Comparação } \\
\text { entre os } \\
\text { Grupos }\end{array}$ \\
\hline G1 e G2 & 771,000 & $-1,568$ & 0,117 & SD* \\
\hline G1 e G3 & 945,000 & $-2,679$ & 0,007 & G1>G3 \\
\hline G1 e G4 & 579,500 & $-3,183$ & 0,001 & G1>G4 \\
\hline G2 e G3 & 1526,000 & $-1,029$ & 0,303 & SD \\
\hline G2 e G4 & 950,500 & $-1,790$ & 0,073 & SD \\
\hline G3 e G4 & 1514,000 & $-1,094$ & 0,274 & SD \\
\hline
\end{tabular}

*SD: Sem Diferenças Estatisticamente Significantes Fonte: Dados da pesquisa.

Os resultados obtidos por meio do teste de Mann-Whitney em relação ao autoconceito pessoal indicaram diferenças estatisticamente significantes entre os grupos G1-G3 e G1-G4. Os alunos isolados apresentaram um autoconceito pessoal mais positivo do que aqueles com rejeição moderada e com alta rejeição entre os seus colegas de classe. Assim como que o verificado na escala familiar, os resultados apresentados na Tabela 3 revelam que o grupo com alta rejeição foi o que teve menor média na escala de autoconceito pessoal.

\section{Discussão e considerações finais}

As relações entre autoconceito e aceitação e rejeição social, no grupo de amigos, foram, até o momento, pouco investigadas pela literatura. No entanto, essas variáveis, individualmente, têm se mostrado frequentemente importantes no estudo do contexto escolar e de suas relações com o desempenho acadêmico de alunos. Um baixo autoconceito tem aparecido associado ao baixo desempenho e às dificuldades de aprendizagem de crianças e adolescentes, enquanto aqueles que apresentam um autoconceito mais elevado estão menos propensos a apresentar esses problemas (Sisto, Urquijo \& Souza, 1999; Lopes, Cruz \& Rutherford, 2002; Stevanato, Loureiro, Linhares \& Marturano, 2003; Saravali, 2004).

Por sua vez, a condição de aceitação ou não da criança ao grupo de amigos da escola também tem se mostrado como uma variável social de importância no âmbito educacional, tendo em vista que a situação de aprendizagem e o contexto escolar pressupõem a interação entre os alunos. Acresce-se a isso o fato de que as crianças que conseguem uma melhor adaptação a esse contexto e que são mais acolhidas pelo grupo também têm apresentado menores possibilidades de integrarem grupos de risco, o que destaca a importância desses dois elementos no contexto escolar e da aprendizagem.

Dessa forma, considerou-se oportuno investigar em que medida essas duas variáveis guardam relações entre si, a fim de que se possa avançar no conhecimento do funcionamento dos sujeitos, tanto do ponto de vista individual quanto de sua inserção no social. Nesse sentido, este estudo revelou interações entre essas duas variáveis que merecem serem destacadas.

Os resultados apontaram para o fato de que houve poucas diferenças significativas entre os grupos de aceitação social no que diz respeito ao autoconceito, e que essas estiveram restritas à subescala escolar, e apenas para alguns grupos. Os estudantes isolados tiveram média mais alta nesta subescala, quando comparados aos demais grupos, e estes se 
diferiram apenas em relação ao grupo de baixa aceitação. A outra diferença encontrada diz respeito ao grupo de aceitação moderada e baixa aceitação, sendo que, neste caso, o primeiro grupo pontuou mais na subescala escolar. De fato, o que se observou neste estudo é que a maior ou menor aceitação pelo grupo de amigos não foi marcada por grandes diferenças no autoconceito dos participantes.

Já no que se refere à rejeição social, observou-se que alguns grupos se diferiram em relação a alguns aspectos do autoconceito, mas também não em todos. Os estudantes com baixa rejeição no grupo de amigos mostraram ter um autoconceito familiar mais positivo do que aqueles com rejeição moderada e com alta rejeição. A influência das interações e situações vivenciadas no contexto familiar, e que puderam ser avaliadas pela subescala de autoconceito familiar, também tem sido apontada por alguns estudos, cujos resultados sugerem a relação entre autoconceito elevado e bom relacionamento com pais e irmãos, enquanto baixos escores de autoconceito aparecem associados a um relacionamento familiar conflituoso (Wentzel \& Feldman, 1996; Sánchez \& Escribano, 1999; Magagnin \& Körbes, 2000).

Diferenças também foram verificadas em relação à subescala de autoconceito pessoal. Os alunos isolados revelaram um autoconceito pessoal mais elevado do que aqueles com rejeição moderada e alta entre os colegas, sendo essas as únicas diferenças encontradas. Neste caso, pode-se dizer que não ser lembrado pelos colegas parece ter um impacto menor sobre o autoconceito pessoal do que ser rejeitado, e que à medida que essa rejeição vai se tornando mais marcada, essas diferenças começam a aparecer.

Os resultados aqui apresentados e as relações verificadas ainda são bastante exploratórios e necessitam ser mais investigados, para que se possa ter uma ideia mais clara a respeito dessas variáveis e dos achados deste estudo. De fato, as relações encontradas não se colocaram, para esta amostra, de forma a permitir nenhuma conclusão, mas apenas a reforçar a ideia de que o autoconceito parece ser mesmo de construção multidimensional, o que significa afirmar que construímos vários autoconceitos sobre nós mesmos e que, portanto, podemos ter um autoconceito escolar, familiar, social e pessoal que diferem entre si, e que possivelmente irão influenciar de forma diferente as relações que estabelecemos com os outros e conosco.
Nesse sentido, vale destacar a necessidade de investigar essa possível multidimensionalidade do autoconceito e até que ponto cada uma dessas construções será capaz de influenciar ou não, a longo prazo, a de outros domínios do autoconceito, bem como de outros aspectos do funcionamento social e individual.

\section{Referências}

Carneiro, G. R. S., Martinelli, S. C., \& Sisto, F. F. (2003). Autoconceito e dificuldades de aprendizagem na escrita. Psicologia: Reflexão e Crítica, 16(3), 427-434.

Coie, J. D., Terry, R., Lenox, K., Lochman, J., \& Hyman, C. (1995). Childhood peer rejection and aggression as predictors of stable patterns of adolescent disorder. Development and Psychopathology, 7(4), 697-713.

Cooley, C. H., \& Ayres, R. R. (1988). Self-concept and success-failure attributions of non-handicapped students and students with learning disabilities. Journal of Learning Disabilities, 21(3), 174-178.

Durrant, J. E., Cunningham, C. E., \& Volker, S. (1990). Academic, social and general self-concepts of behavioral subgroups of learning disabled children. Journal of Educational Psychology, 82 (4), 657-663.

Jackson, L. D., \& Bracken, B. A. (1998). Relationship between students' social status and global domain-specific self-concepts. Journal of School Psychology, 36(2), 233-246.

Jacob, A. V. (2001). 0 desempenho escolar e suas relações com o autoconceito e auto-eficácia. Tese de Doutorado, Universidade de São Paulo, Ribeirão Preto.

Lee-Manoel, C. L., Morais, M. L. S., Bussab, V. S. R., \& Otta, E. (2002). Quem é bom (e eu gosto) é bonito: Efeitos da familiaridade na percepção de atratividade física em pré-escolares. Psicologia Reflexão e Crítica, 15(2), 271-282.

Lopes, J., Cruz, C., \& Rutherford Jr., R. B. (2002). The relationship of peer perceptions to student achievement and teacher ratings of 5 th and 6 th grade students. Education and Treatment of Children, 25(4), 476-495.

Magagnin, C., \& Körbes, J. M. (2000). Autoconceito do adolescente: Relacionamento familiar e limites. Aletheia, 12, 65-81. 
Martinelli, S. C., \& Schiavoni, A. (2009). Percepção do aluno sobre sua interação com o professor e status sociométrico. Estudos de Psicologia, 26(3), 327-336.

Morais, M. L. S., Otta, E., \& Scala, C. T. (2001). Status sociométrico e avaliação de características comportamentais: Um estudo de competência social em pré-escolares. Psicologia: Reflexão e Crítica, 14(1), 119-131.

Newcombe, N. (1999). Desenvolvimento infantil: Abordagem de Mussen. (Cláudia Buchweitz, Trad.). Porto Alegre: Artes Médicas.

Okano, C. B., Loureiro, S. R., Linhares, M. B. M., \& Marturano, E. M. (2004). Crianças com dificuldades escolares atendidas em programa de suporte psicopedagógico na escola: Avaliação do autoconceito. Psicologia: Reflexão e Crítica, 17(1), 121-128.

Rothman, H. R., \& Cosden, M. (1995). The relationship between self-perception of a learning disability and achievement, self-concept and social support. Learning Disability Quarterly, 18(3), 203-212.

Rudolph, K. D. (1995). Cognitive representations of self, family, and peers in school-age children: Links with social competence and sociometric status. Child Development, 66(5), 1385-1402.

Sánchez, A. V., \& Escribano, E. A. (1999). Medição do autoconceito. Bauru: EDUSC.

Saravali, E. G. (2004). As relações interpessoais dos alunos com problemas no aprendizado: um estudo a partir do referencial Moreniano. Revista Digital UMBRAL 2000, 16. Recuperado em 14 jan. de 2007, em http: //www.reduc.cl

Silva, V. R. M. G., Löhr, S. S. (2001). Indicadores de rejeição em grupo de crianças. Interação em Psicologia, 5, 9-30.
Sisto, F. F. (2003). Rejeição entre colegas e agressividade na infância. Cadernos de Psicologia, 13(1), 83-98.

Sisto, F. F. (2005). Aceitação-rejeição para estudar e agressividade na escola. Psicologia em Estudo, 10(1), 117-125.

Sisto, F. F., Urquijo, S., \& Souza, M. T. C. C. (1999). Peer acceptance and cognitive development. Psychological Reports, 84(2), 611-616.

Sisto, F. F., \& Martinelli, S. C. (2004). Escala de autoconceito infanto-juvenil (EAC-IJ). São Paulo: Vetor.

Sisto, F. F., \& Martinelli, S. C. (2006). Afetividade e dificuldades de aprendizagem: Uma abordagem psicopedagógica. São Paulo: Vetor.

Stevanato, I. S., Loureiro, S. R., Linhares, M. B. M., \& Marturano, E. M. (2003). Autoconceito de crianças com dificuldades de aprendizagem e problemas de comportamento. Psicologia em Estudo, 8(1), 67-76.

Suehiro, A. C. B. (2006). Autoconceito e desempenho acadêmico em alunos de Psicologia. Psicologia Argumento, 24(44), 55-64.

Tonelotto, J. M. F. (2002). Aceitação e rejeição: Percepção de escolares desatentos no ambiente escolar. Psicologia Escolar e Educacional, 6(2), 141-148.

Urquijo, S. (2002). Auto-concepto y desempeño académico en adolescentes. Relaciones con sexo, edad e institución. Psico USF, 7(2), 211-218.

Wentzel, K. R., \& Feldman, S. S. (1996). Relation of cohesion and power in family dyads to social and emotional adjustment during early adolescence. Journal of Research on Adolescence, 6(2), 225-244. 
